\title{
Skin Lesion as the First Sign of Pulmonary Neoplasia: A Report of 2
}

\section{Cases}

\author{
Eduardo Esteban-Zubero, ${ }^{1, *}$ \\ ${ }^{1}$ Emergency Department Hospital San Pedro, La Rioja, Spain \\ "Corresponding author: Eduardo Esteban-Zubero, Calle Piqueras 98. 26006, Logroño, Spain. Tel: +34-941298000, E-mail: eezubero@gmail.com
}

Received 2015 December 01; Accepted 2016 January 01.

\begin{abstract}
Skin lesions are common clinical phenomena in primary care, as well as emergency situations. Several studies have shown that every patient has an average of 1.7 visits to primary care physicians due to dermatological diseases. These visits can be related to seemingly mild diseases, such as contact dermatitis as the most common disorder in daily clinical practice, or potentially serious diseases, such as melanoma and metastasis. If a primary tumor is diagnosed, skin metastasis can be detected in $20 \%$ to $60 \%$ of patients. Cutaneous metastasis from lung tumor is not common; however, it can lead to a fatal prognosis with a median survival of 5.75 months. The purpose of this study was to highlight the importance of skin lesions as a diagnostic marker of serious disorders such as neoplasm. For this purpose, we conducted a review of skin metastasis and its relationship with different types of cancer, especially pulmonary cancer.
\end{abstract}

Keywords: Skin, Metastasis, Lung Cancer

\section{Introduction}

Skin lesions are common clinical phenomena in primary care, as well as emergency situations. Several studies have shown that every patient has an average of 1.7 visits to primary care physicians due to dermatological diseases (1). These diseases range from seemingly trivial conditions, such as contact dermatitis as the most common disease in daily clinical practice (2), to potentially serious diseases, such as melanoma.

According to previous research, $63 \%$ of patients with melanoma were evaluated by a physician for consultation in the year before diagnosis (1). The purpose of this study was to highlight the importance of skin lesions as a diagnostic marker of serious diseases such as neoplasm. Accordingly, we conducted a review of skin metastasis and its relationship with different types of cancer, especially pulmonary cancer.

\section{Case Presentation}

The first case is a 65-year-old man with a history of tuberculosis, chronic obstructive pulmonary disease (emphysema type), dyslipidemia, and smoking for 10 years (40 cigarettes per day 20 years ago). The patient received treatment with tiotropium, inhaled budesonide-formoterol, as- pirin, fluvastatin, manidipine, and omeprazole. He presented with a papular and erythematous lesion in the abdomen $(2 \times 2 \mathrm{~cm})$ at 2 months before admission (Figure 1) without any pain or itching. Concomitantly, an increase in dyspnea, cough, and yellow sputum was observed in the month before admission, which was sometimes accompanied by slightly bloody sputum. Furthermore, hyperoxia, fatigue, and weight loss were observed in the last 2 months.

Cardiac and lung explorations were found to be normal. There was no tenderness of spinous processes at any level, while tenderness was found in the right paraspinal musculature in the D4-D8 region due to muscle spasm. The abdomen showed a papular and erythematous lesion, without trophic changes or increase in temperature. Neurological examination was normal in the patient. Analytical laboratory tests revealed discrete leukocytosis $(16,900$ $/ \mathrm{mm}^{3}$ ) with an increase in neutrophil count (84\%). Thoracic $\mathrm{X}$-ray indicated left-sided laminar atelectasis. Considering the changes in the patient's condition and symptoms, a thoracoabdominal scan was performed, showing a mass (diameter, $4 \mathrm{~cm}$ ) in the posterior segment of the left upper lobe, contacting the visceral pleura and oblique fissure with mediastinal lymphadenopathy and bilateral pleural effusion, mainly in the left lung.

In addition, D5 bone metastases and soft tissue tumor, protruding into the thoracic cavity with possible spinal in- 
Figure 1. The Papular and Erythematous Lesion in the Abdomen $(2 \times 2 \mathrm{~cm})$
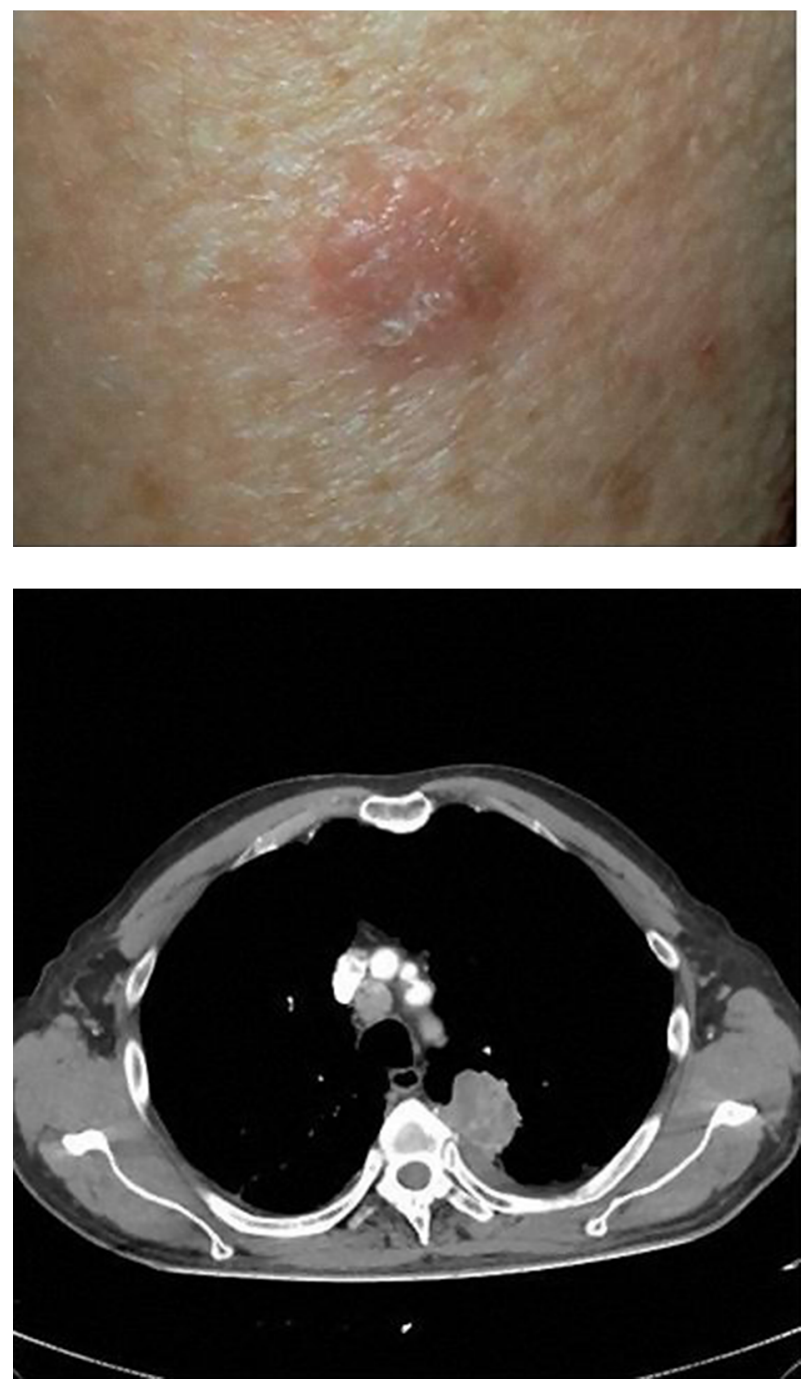

The thoracoabdominal scan showed a mass (diameter, $4 \mathrm{~cm}$ ), located in the posterior segment of the upper left lobe contacting the visceral pleura and oblique fissure with mediastinal lymphadenopathy and bilateral pleural effusion, mainly in the left lung. D5 bone metastasis and soft tissue tumor, protruding into the thoracic cavity with possible spinal involvement, were also observed.

volvement, were observed. Based on these findings, diagnosis of stage-4 pulmonary neoplasia (T2 N3 M1) was established (Figure 1). Biopsy of lung injury showed a large cell carcinoma, which was consistent with the biopsy of skin lesion indicating a large-cell (undifferentiated) adenocarcinoma.

The second case was a 54-year-old man with a history of active smoking (40 cigarettes per day during 15 years). He presented with 2 papular lesions (one on the left collarbone and one on the right scapula) in the past month. In addition, he reported coughing in the past several months with brownish sputum and some blood in the last month. No dyspnea or other alterations were observed.

Cardiac, lung, and abdominal explorations were normal. At the vertebral level, no tenderness or change in the accessory muscles was observed. Neurological examination was also normal. Physical examination revealed 2 papular lesions, one on the left collarbone and one on the right scapula (diameter, $5 \mathrm{~cm}$ ) with hard consistency without any disturbances.

Analytical laboratory tests revealed discrete leukocytosis $\left(20,300 / \mathrm{mm}^{3}\right)$ without any changes in the formula. Chest X-ray showed an $18.5-\mathrm{mm}$ nodule with left apical suprahilar hilum traction. In the thoracoabdominal scan, a tumor (diameter, $45 \mathrm{~mm}$ ) in the left pulmonary hilum, encompassing the main bronchus, was observed. In addition, segment- 6 bronchus indicated stenosis of the lower lobe bronchus. Mediastinal lymph nodes, as well as a metastatic tumor (diameter, $55 \mathrm{~mm}$ ) in the left adrenal gland, were also observed.

Finally, left costovertebral joint destruction at level T9 was described, producing soft tissue mass effects and bulges on the lung parenchyma. Based on these findings, diagnosis of early stage-4 lung neoplasia (T4 N2 M1) was confirmed (Figure 2). Biopsy of the skin lesion indicated poorly differentiated metastatic squamous cell carcinoma (Figure 2).

\section{Discussion}

In 2012, the incidence of lung cancer was estimated at 1.8 million, causing 1.6 million deaths (3). The prognosis of lung cancer is poor with a 5-year survival of approximately $15 \%$. The most common histological type is adenocarcinoma, followed by squamous cell carcinoma, small cell carcinoma, and large cell carcinoma (4). It often metastasizes to the hilar lymph nodes, adrenal glands, liver, brain, and bones and is sometimes accompanied by rare cases of cutaneous metastasis (5). Although the latter is rare, it is an important sign to suspect a primary lung injury due to the difficult diagnosis of this pathology. In general, skin lesions are the initial manifestation of internal malignancies in $0.8 \%$ of cases; however, they indicate a very advanced stage of the disease and a poor prognosis if detected (6).

Considering gender, significant differences were found in the etiology of skin metastasis. In men, skin metastases are mainly due to primary malignant lung tumors (12\% - 28\%), gastrointestinal tumors (11\% - 19\%), and melanoma (13\% - 32\%). On the other hand, in women, the lung is the fifth most common origin of tumor (4\%), following breasts (69\%), large intestine (9\%), melanoma (5\%), and ovaries (4\%). Importantly, skin metastases are 

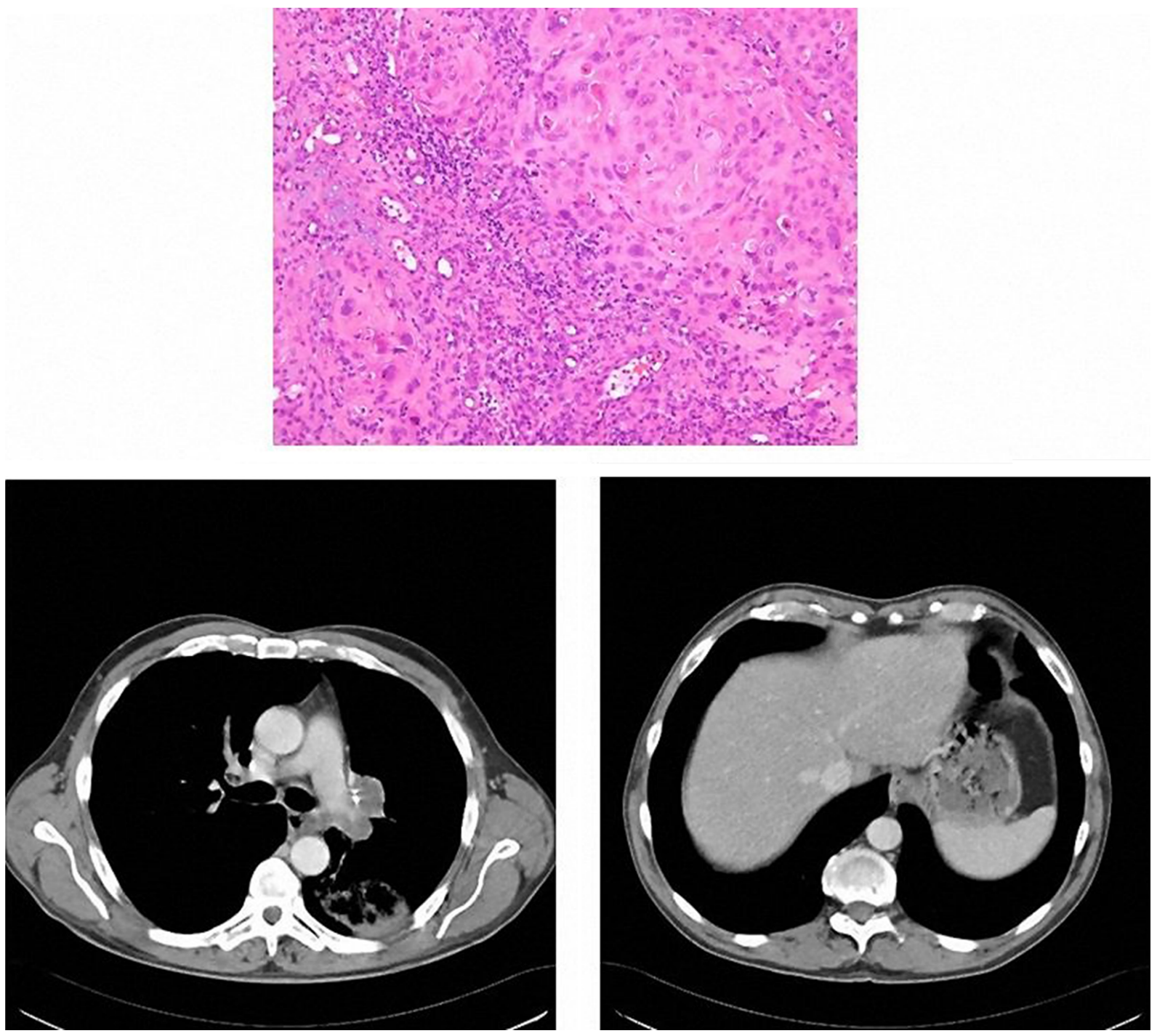

The thoracoabdominal scan showed a tumor (diameter, $45 \mathrm{~mm}$ ) in the left pulmonary hilum encompassing the main bronchus. Segment-6 bronchus indicated stenosis in the lower lobe bronchus with mediastinal lymph nodes and showed a metastatic tumor (diameter, $55 \mathrm{~mm}$ ) on the left adrenal gland. T9 left costovertebral joint destruction was described, producing soft tissue mass effects and bulges on the lung parenchyma.

detected in $20 \%$ to $60 \%$ of patients with a diagnosis of primary tumor (7).

The percentage of patients with primary lung metastases who develop skin cancer ranges from $1 \%$ to $12 \%$. Although lung cancer does not often metastasize to the skin, the median survival time is 5.75 months in case of occurrence (8). These metastases are often poorly differentiated from histopathological conditions (9) and can invade the lymphovascular system; however, they are usually limited to the dermis and subcutaneous layers of the skin (10).

Cutaneous metastases are rare in lung cancer, but if present at diagnosis, they indicate a poor prognosis. Therefore, accurate medical history-taking is necessary to detect the warning signs, as factors such as weight loss and hemoptysis are extremely important in these patients to assess the relevance of skin lesions.

\section{Footnotes}

Authors' Contribution: All the authors approved the final manuscript and actively contributed to its development. 
Conflict of Interest: The authors declare no conflicts of interest related to this article.

Funding/Support: The authors declare no external funding sources or comments were received.

\section{References}

1. Goulart JM, Quigley EA, Dusza S, Jewell ST, Alexander G, Asgari MM, et al. Skin cancer education for primary care physicians: a systematic review of published evaluated interventions. J Gen Intern Med. 2011;26(9):1027-35. doi: 10.1007/s11606-011-1692-y. [PubMed: 21472502].

2. Fleischer AJ, Herbert CR, Feldman SR, O'Brien F. Diagnosis of skin disease by nondermatologists. Am J Manag Care. 2000;6(10):1149-56. [PubMed: 11184670].

3. Brambilla E, Travis WD. World cancer report. Geneva, Switzerland: World Health Organization; 2014

4. Novaes FT, Cataneo DC, Ruiz Junior RL, Defaveri J, Michelin OC, Cataneo AJ. Lung cancer: histology, staging, treatment and survival. J Bras Pneumol. 2008;34(8):595-600. [PubMed: 18797744].
5. Perisano C, Spinelli MS, Graci C, Scaramuzzo L, Marzetti E, Barone C, et al. Soft tissue metastases in lung cancer: a review of the literature. Eur Rev Med Pharmacol Sci. 2012;16(14):1908-14. [PubMed: 23242715].

6. Altintoprak F, Baytekin HF, Tasdemir C. Primary small cell carcinoma of the lung presenting with breast and skin metastases. Korean J Intern Med. 2011;26(2):207-9. doi: 10.3904/kjim.2011.26.2.207. [PubMed: 21716912].

7. Lookingbill DP, Spangler N, Sexton FM. Skin involvement as the presenting sign of internal carcinoma.J Am Acad Dermatol.1990;22(1):1926. doi: 10.1016/0190-9622(90)70002-y. [PubMed: 2298962].

8. Molina Garrido MJ, Guillen Ponce C, Soto Martinez JL, Martinez YC, Carrato Mena A. Cutaneous metastases of lung cancer. Clin Transl Oncol. 2006;8(5):330-3. [PubMed: 16760007].

9. Kamble R, Kumar L, Kochupillai V, Sharma A, Sandhoo MS, Mohanti BK. Cutaneous metastases of lung cancer. Postgrad Med J. 1995;71(842):741-3. [PubMed: 8552539].

10. Saeed S, Keehn CA, Morgan MB. Cutaneous metastasis: a clinical, pathological, and immunohistochemical appraisal. J Cutan Pathol. 2004;31(6):419-30. doi: 10.1111/j.0303-6987.2004.00207.x. [PubMed: 15186430]. 